\section{kompakt}

\section{Kinderimpfung: Meistens befürwortet}

Was die Fürsorge für den Nachwuchs in puncto impfen angeht, befürworten die meisten Eltern die Impfungen im Kindesalter. So haben $64 \%$ der befragten 3000 Eltern keine Vorbehalte gegen die Prophylaxe, 35\% stehen einzelnen Impfungen kritisch gegenüber und nur $1 \%$ lehnen sie vollständig ab.

Ergebnisbericht BZgA, Mai 2011

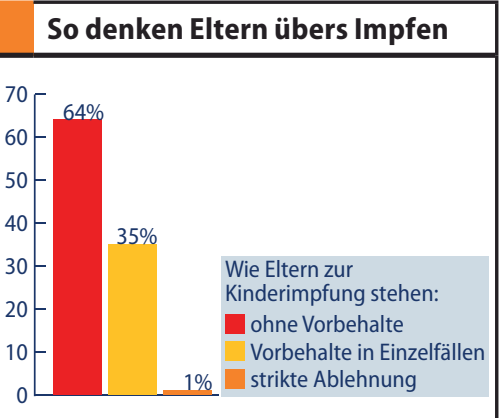

\section{Bandscheibenersatz: besser als Reha?}

Bei chronischen Lumbalgien statt Reha gleich unters Messer? Die Ergebnisse einer Studie mit 173 Teilnehmern im Alter von 22 bis 55 Jahren sprechen dafür. Demnach erzielten die operierten Patienten langfristig bessere Ergebnisse als diejenigen, die sich aufgrund der Rückenschmerzen einer 60-stündigen Rehabilitationstherapie unterzogen. Experten warnen jedoch vor der leichtfertigen Implantation einer Bandscheibenprothese: Die Risken einer Op. dürften nicht unterschätzt werden. Hellum C et al, BMJ 2011, 342:d2786

\section{springermedizin.de via Twitter folgen}

Der Sommer ist da und das
Zwitschern geht immer weiter:
Werden Sie zum „Follower" und
"lauschen“ Sie uns bei Twitter.
twitter.com/springermedizin

\title{
Trigger Verkehrsunfall
} Vom Crash zum Schmerzsyndrom

Verkehrsunfälle, nicht aber andere traumatische Ereignisse scheinen bei prädisponierten Personen ein Trigger für chronische ausgedehnte Schmerzsyndrome zu sein.

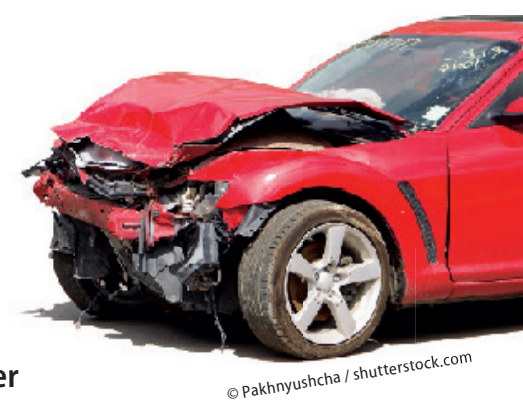

Darauf weist eine prospektive populationsbasierte Kohortenstudie hin, in der über einen Zeitraum von vier Jahren dreimal Schmerzen des Bewegungsapparats und parallel auftretende psychologische Stressoren ermittelt wurden. Dabei fragten die Untersucher nach sechs körperlichen Traumen: Verkehrsunfall, Unfall am Arbeitsplatz, Operation, Knochenbruch, Krankenhausaufenthalt oder Geburt.

\section{Keine unmittelbare Verletzungsfolge}

Von den Studienteilnehmern entwickelten im Studienzeitraum 12\% chronische Ganzkörperschmerzen („chronic widespread pain“), definiert nach der American Academy of Rheumatology (ACR) als Schmerzen sowohl oberals auch unterhalb der Gürtellinie, auf der rechten wie der linken Körperseite und über mindestens drei Monate anhaltend. Mehr als ein Drittel der Betroffenen berichtete von einem oder mehreren traumatischen Ereignissen vor Beginn der Schmerzen. Speziell Verkehrsunfälle erhöhten das Risiko für chronische Ganzkörperschmerzen um 84\%, während Krankenhausaufenthalt, Operation oder Geburt keinen Einfluss auf dieses Risiko hatten. Die Autoren vermuten, dass bestimmte Personen bereits physisch oder psychisch prädisponiert sind und der Verkehrsunfall nur ein Trigger ist, der die chronischen Ganzkörperschmerzen auslöst, es sich also nicht um physische Unfallfolgen handelt. Weitere Untersuchungen müssen zeigen, was gerade den Verkehrsunfall zu einem Auslöser chronischer Ganzkörperschmerzen macht.

(FK) Jones GT et al, Arthritis Care Res 2011, 5:696

\section{Hurra! Spermien wieder auf dem Vormarsch Europa doch nicht vom Aussterben bedroht}

\section{Männer können aufatmen: Die soge- nannte „Fertilitätskrise“ mit Rückgang der Spermienqualität scheint vorbei zu sein. Eine Untersuchung belegte, dass sich die Konzentration im Ejakulat auf einen Mittelwert eingependelt hat.}

In Studien aus den 90er Jahren hatten Forscher Alarm geschlagen: Der Rückgang der Spermienkonzentration- und Qualität von Männern aus Industrienationen schien unaufhaltsam. Als Ursa- che wurde eine zunehmende Exposition mit Umweltchemikalien postuliert.

Diese Ergebnisse widerlegt eine Untersuchung an mehr als 5000 Ejakulaten von dänischen Rekruten. Hier zeigte sich, dass die Spermienkonzentration zwischen 1996 und 2010 um einen Mittelwert von 40-45 Mio./ml schwankte. Eine schwedische Studie zog eine ähnliche Bilanz, eine finnische Untersuchung konnte die Befunde jedoch nicht bestätigen. (cd) Dtsch Arztebl 07.06.2011 